\title{
Analytic confinement: the glueball and meson masses, and the weak decay constants
}

\author{
Gurjav Ganbold*† \\ Bogoliubov Laboratory of Theoretical Physics, \\ Joint Institute for Nuclear Research, 141980 Dubna, Russia \\ E-mail: ganboldethsun1.jinr.ru
}

\begin{abstract}
Independent and analytic estimates of the two-quark and two-gluon bound states have been performed within a relativistic quantum-field model in the presence of analytic confinement. The ladder Bethe-Salpeter equation is solved for the spectra of two-gluon and two-quark bound states by involving a minimal set of physical parameters (the quark masses, the coupling constant and the confinement scale). The model provides a reasonable framework to compute data simultaneously in three sectors of low-energy particle physics, namely, the lowest glueball mass, the conventional meson masses (from $\pi(140)$ up to $\Upsilon(9460)$ ), and the pion and kaon weak decay constants.
\end{abstract}

8th Conference Quark Confinement and the Hadron Spectrum September 1-6 2008

Mainz, Germany

\footnotetext{
* Speaker.

${ }^{\dagger}$ Institute of Physics and Technology, 210651 Ulaanbaatar, Mongolia
} 


\section{Introduction}

The color confinement in QCD is an explanation of the physics phenomenon that color charged particles are not observed. However, there is no analytic proof that QCD should be color confining, the reasons for quark confinement may be somewhat complicated. There exist different suggestions about the origin of confinement, e.g., [1]. One of the earliest suggestion is the Analytic Confinement (AC) based on the assumption that the QCD vacuum is realized by the self-dual vacuum gluon field [2]. Particularly, a nonzero self-dual homogenous gluon field $B_{\mu}(x)$ with constant strength provides the vacuum of the quark-gluon system and makes the quark and gluon propagators entire analytic functions [3]. However, direct use of these propagators encounters complex formulae and cumbersome estimations. On the other hand, the conventional perturbation theory cannot be used at low energy, where the most interesting and novel behavior is expected. Different nonperturbative approaches have been proposed to deal with the long distance properties of QCD, such as chiral perturbation theory, QCD sum rule, heavy quark effective theory, lattice QCD simulations, coupled Schwinger-Dyson equation model etc. Along outstanding advantages these approaches have also obvious shortcomings. Dealing with the two body relativistic bound state problems one should use a relativistic approach, e.g., the Bethe-Salpeter equation (BSE) [4].

The calculations of hadron mass characteristics on the level of experimental data precision still remain among the unsolved problems in QCD due to some technical and conceptual difficulties. In such a case, it is useful to investigate the corresponding low-energy effective theories and phenomenological models. Earlier we studied a 'toy' model of interacting scalar 'quarks' and 'gluons' that demonstrated a qualitatively correct description of the mass spectrum of the two- and threeparticle bound states [5,6]. Below we extend this investigation by taking into account the spin, color and flavor degrees. Our goal is to suggest a model describing hadrons as relativistic bound states in terms of quarks and gluons and to calculate simultaneously the mass spectra of light and heavy mesons, the decay constants $f_{\pi}$ and $f_{K}$, and the lowest glueball mass.

\section{The model}

Because of the complexity of QCD, it is often prudent to examine simpler systems exhibiting similar characteristics first. Consider a simple relativistic quantum-field model of quark-gluon interaction assuming that the AC takes place. The model Lagrangian reads[7]:

$$
\mathscr{L}=-\frac{1}{4}\left(F_{\mu v}^{A}-g f^{A B C} \mathscr{A}_{\mu}^{B} \mathscr{A}_{v}^{C}\right)^{2}+\sum_{f}\left(\bar{q}_{f}^{a}\left[\gamma_{\alpha} \partial_{\alpha}-m_{f}+g \Gamma_{\alpha}^{C} \mathscr{A}_{\alpha}^{C}\right]^{a b} q_{f}^{b}\right)
$$

where $\mathscr{A}_{\alpha}^{C}$ - gluon field, $F_{\mu \nu}^{A}=\partial_{\mu} \mathscr{A}_{v}^{A}-\partial_{v} \mathscr{A}_{\mu}^{A}, q_{f}^{a}$ - quark triplet with color $a$, flavor $f$ and mass $m_{f} ; g$ - the coupling strength and $\Gamma_{\alpha}^{C}=i \gamma_{\alpha} t^{C}$.

We restrict the consideration within the ladder BSE that is sufficient to estimate the spectra of two-quark and two-gluon bound states with reasonable accuracy $[6,7]$. The path integrals defining the leading-order contributions to the two-quark and two-gluon bound states read:

$$
\begin{aligned}
& Z_{q \bar{q}}=\iint \mathscr{D} \bar{q} \mathscr{D} q \exp \left\{-\left(\bar{q} S^{-1} q\right)+\frac{g^{2}}{2}\langle(\bar{q} \Gamma \mathscr{A} q)(\bar{q} \Gamma \mathscr{A} q)\rangle_{D}\right\}, \\
& Z_{\mathscr{A} \mathscr{A}}=\left\langle\exp \left\{-\frac{g}{2}(f \mathscr{A} \mathscr{A} F)\right\}\right\rangle_{D}, \quad\langle(\bullet)\rangle_{D}=\int \mathscr{D} \mathscr{A} e^{-\frac{1}{2}\left(\mathscr{A} D^{-1} \mathscr{A}\right)}(\bullet) .
\end{aligned}
$$


The structure of the QCD vacuum is not well established and one encounters difficulties by defining the explicit quark and gluon propagator at the confinement scale. The Green's functions in QCD are tightly connected to confinement and are ingredients for hadron phenomenology. On the other hand, the matrix elements of hadron processes are integrated characteristics of the propagators and vertices. Therefore, taking into account the correct global symmetry properties and their breaking by introducing additional physical parameters may be more important than the working out in detail. Consider the quark and gluon (in Feynman gauge) propagators:

$$
\begin{aligned}
& \tilde{S}_{\mu \nu}^{a b}(\hat{p})=\delta^{a b}\left\{\frac{i \hat{p}}{m_{f}^{2}+\Lambda m_{f}}+\frac{1 \pm \gamma_{5} \omega\left(m_{f} / \Lambda\right)}{m_{f}}\right\}_{\mu \nu} \exp \left\{-\frac{p^{2}+m_{f}^{2}}{2 \Lambda^{2}}\right\} \\
& \tilde{D}_{\mu v}^{A B}(p)=\delta_{\mu v} \delta^{A B} \frac{1}{p^{2}} \exp \left(-p^{2} / 4 \Lambda^{2}\right)
\end{aligned}
$$

where $\hat{p}=p_{\mu} \gamma_{\mu}$ and $\omega(z)=\left(1+z^{2} / 4\right)^{-1}$. These entire analytic functions in Euclidean space represent approximations to the propagators calculated in [3]. The sigh \pm in the quark propagator means the self- and antiself-dual modes of the vacuum fields. The model parameters are: the coupling constant $\alpha_{s} \doteq g^{2} / 4 \pi$, the confinement scale $\Lambda$ and the quark masses $\left\{m_{u}=m_{d}, m_{s}, m_{c}, m_{b}\right\}$.

Further we allocate the one-gluon exchange term between colored bi-quark currents and isolate the color-singlet combination. Then, we perform a Fierz transformation $\left(i \gamma_{\mu}\right) \delta^{\mu v}\left(i \gamma_{v}\right)=\sum_{J} C_{J}$. $O_{J} O_{J}$ for $O_{J} \doteq\left\{I, i \gamma_{5}, i \gamma_{\mu}, \gamma_{5} \gamma_{\mu}, \frac{i}{2}\left[\gamma_{\mu}, \gamma_{v}\right]\right\}$ and pass to relative co-ordinates in the center-of-mass system. By introducing a system of orthonormalized functions $\left\{U_{N}\right\}$ and expanding the bi-quark nonlocal current on the basis we can perform explicit integration over the quark fields and rewrite path integral (2.2) in terms of auxiliary meson fields $B_{N}(x)$

$Z_{q \bar{q}} \rightarrow Z=\int \prod_{N} \mathscr{D} B_{N} \exp \left\{-\frac{1}{2}\left(B_{N}\left[1+\frac{4 g^{2} C_{J}}{9}\left(\sqrt{D} U_{N} \operatorname{Tr}\left[O_{J} S O_{J} S\right] \sqrt{D} U_{N}\right)\right] B_{N}\right)-W_{\text {res }}\left[B_{N}\right]\right\}$,

where $W_{\text {res }}\left[B_{N}\right]$ describes the interaction between mesons. We introduce an hadronization Ansatz and identify $B_{N}(x)$ with mesons carrying quantum numbers $N=\left\{J, \ell, n_{r}, f_{1}, f_{2}\right\}$.

Meson ground states. Note, the diagonalization of the kinetic part on the basis $\left\{U_{N}\right\}$ is equivalent to the solution of the ladder BSE:

$$
\frac{4 g^{2} C_{J}}{9}\left(\sqrt{D} U_{N} \operatorname{Tr}\left[O_{J} S O_{J} S\right] \sqrt{D} U_{N}\right)=\delta_{N N^{\prime}} \lambda_{N}\left(-p^{2}\right),
$$

where the polarization kernel reads

$$
\lambda_{N}\left(-p^{2}\right) \doteq \frac{4 g^{2} C_{J}}{9} \iint d x d y \sqrt{D(x)} \int \frac{d^{4} k}{(2 \pi)^{4}} e^{-i k(x-y)} \operatorname{Tr}\left[O_{J} \tilde{S}\left(\hat{k}+\xi_{1} \hat{p}\right) O_{J} \tilde{S}\left(\hat{k}-\xi_{2} \hat{p}\right)\right] \sqrt{D(y)} .
$$

It is convenient to use the renormalized form:

$$
\left(U_{N}\left[1+\lambda_{N}\left(-p^{2}\right)\right] U_{N}\right)=\left(U_{N}\left[1+\lambda_{N}\left(M_{N}^{2}\right)-\dot{\lambda}_{N}\left(M_{N}^{2}\right)\left[p^{2}+M_{N}^{2}\right] U_{N}\right) \doteq\left(U_{R}\left[p^{2}+M_{N}^{2}\right] U_{R}\right) .\right.
$$

In relativistic quantum field theory a stable bound state of $n$ massive particles shows up as a pole in the S-matrix with a center of mass energy. Accordingly, the meson mass may be derived from equation [7]:

$$
1+\lambda_{N}\left(M_{N}^{2}\right)=0, \quad p^{2}=-M_{N}^{2}
$$


Below we consider the meson ground states $\left(\ell=0, n_{r}=0\right)$, the pseudoscalar $\left(P: J^{P C}=0^{-+}\right)$ and vector $\left(V: J^{P C}=1^{--}\right)$mesons. The quark constituent masses $\left\{m_{u d}, m_{s}, m_{c}, m_{b}\right\}$ and coupling constant $\alpha_{s}$ have been fixed to fit exactly the meson masses $M_{\pi}(138 \mathrm{MeV}), M_{\rho}(770), K(495)$, $J / \Psi(3097)$ and $\Upsilon(9460)$ while $\Lambda$ has been estimated to describe reasonably the weak decay constants $f_{\pi}$ and $f_{K}$ (see below). Finally, we fix the following values of model parameters:

$$
\begin{array}{ll}
\alpha_{s} & =0.53114, \quad \Lambda=413.1 \mathrm{MeV}, \\
m_{s} & =301.2 \mathrm{MeV}, \quad m_{c d}=228.5 \mathrm{MeV}, \\
m_{c}=1305.3 \mathrm{MeV}, & m_{b}=4608.1 \mathrm{MeV} .
\end{array}
$$

By using these parameters we calculate mass spectra of the pseudoscalar and vector mesons given in Fig. 1 in comparison with experimental data from PDG-2006. The relative error of our estimate does not exceed $\sim 4$ per cent in the whole range of meson masses (from $140 \mathrm{MeV}$ up to $9.5 \mathrm{GeV}$ ). For $\omega$ and $\Phi$ mesons we use the quark-flavor basis mixing scheme with angle $\theta_{V}=76.5^{\circ}$. Note,

\begin{tabular}{|c|c|c|c|c|c|c|c|}
\hline$J^{P C}=0^{-+}$ & $M_{\mathbf{P}}$ & $J^{P C}=0^{-+}$ & $M_{\mathbf{P}}$ & $J^{P C}=1^{--}$ & $M_{\mathbf{V}}$ & $J^{P C}=1^{--}$ & $M_{\mathbf{V}}$ \\
\hline$\pi(138)$ & 138 & $\eta_{c}(2979)$ & 3017 & $\rho(770)$ & 770 & $D_{s}^{*}(2112)$ & 2063 \\
$K(495)$ & 495 & $B(5279)$ & 5450 & $\omega(782)$ & 776 & $J / \Psi(3097)$ & 3097 \\
$\eta(547)$ & 547 & $B_{s}(5370)$ & 5529 & $K^{*}(892)$ & 899 & $B^{*}(5325)$ & 5577 \\
$D(1870)$ & 1855 & $B_{c}(6286)$ & 6462 & $\Phi(1019)$ & 1017 & $\Upsilon(9460)$ & 9460 \\
$D_{s}(1970)$ & 1964 & $\eta_{b}(9300)$ & 9435 & $D^{*}(2010)$ & 1954 & & \\
\hline
\end{tabular}

Table 1: Estimated masses of the ground-state mesons compared with experimental data (in units of MeV). the value $\alpha_{s}=0.53114$ is obtained to fit the $\pi$ meson mass, therefore, it is in agreement with the prediction of the QCD running coupling $\alpha_{Q C D} \sim 0.4 \div 0.7$ in the low-energy region (see, e.g., [8]).

Weak decay constants. An important quantity in the meson physics is the weak decay constant. For the pseudoscalar mesons $f_{P}$ is defined by the following current-meson duality formula

$$
i f_{P} p_{\mu}=\left\langle 0\left|J_{A}(0)\right| U_{R}(p)\right\rangle,
$$

where $J_{A}$ is the axial vector part of the weak current. Accordingly, we estimate

$$
i f_{P} p_{\mu}=\frac{\sqrt{2} g}{3} \int \frac{d k}{(2 \pi)^{4}} \int d x e^{-i k x} U_{R}(x) \sqrt{D(x)} \operatorname{Tr}\left\{i \gamma_{5} \tilde{S}\left(\hat{k}+\xi_{1} \hat{p}\right) \gamma_{5} \gamma_{\mu} \tilde{S}\left(\hat{k}-\xi_{2} \hat{p}\right)\right\} .
$$

With optimal values for $\left\{m_{u}, m_{s}, \alpha_{s}, \Lambda\right\}$ (2.6) we calculate the weak decay constants of light mesons

$$
f_{\pi}=117 \mathrm{MeV}, \quad f_{K}=178 \mathrm{MeV} .
$$

Compare our estimates with the latest data $[9,10]$

$$
f_{\pi}=130.7 \pm 0.1 \pm 0.36 \mathrm{MeV}, \quad f_{K}=159.8 \pm 1.4 \pm 0.44 \mathrm{MeV} .
$$

Glueball lowest state. Glueballs are the most unusual particles predicted by the QCD but not found experimentally yet [11]. Pure two-gluon bound states are the most studied gluonic systems in the literature. Recent lattice calculations, QCD sum rules, 'tube' and constituent glue models predict that the lightest glueball has quantum number $J^{P C}=0^{++}$(scalar) [12]. In quenched lattice QCD the lightest glueball is a scalar object with a mass of (1.66 \pm 0.05$) \mathrm{GeV}$ [13]. 
Consider a two-gluon scalar bound state within ladder BSE method. By omitting details of similar intermediate calculations (see previous section) we obtain the final analytic expression for the lowest-state glueball mass:

$$
M_{G}=2 \Lambda\left[\ln \left(\frac{\alpha_{c r i t}}{\alpha_{s}}\right)\right]^{1 / 2}, \quad \alpha_{c r i t} \doteq \frac{3 \pi(3+2 \sqrt{2})^{2}}{8} .
$$

Our purpose is to describe simultaneously different sectors of low-energy particle physics. Therefore, we use the parameters $\left(\alpha_{s}=0.53114, \Lambda=413.1 \mathrm{MeV}\right)$ optimal for the meson groundstate spectra (2.6) and weak decay constants (2.8). With these parameters we calculate the loweststate (scalar) glueball mass

$$
M_{G}=1718 \mathrm{MeV}
$$

Our estimate (2.10) is in reasonable agreement with prediction $1750(50)(80) \mathrm{MeV}$ for the mass of the lightest glueball from quenched QCD [14]. The most recent quenched lattice estimate with improved action favors a scalar glueball mass close to $M_{G}=1710 \pm 50 \pm 58 \mathrm{MeV}$ [15].

In conclusion, we have considered a simple relativistic quantum field model of interacting quarks and gluons confined analytically. Our guess about the structure of the quark-gluon interaction in the confinement region was proved to be correct and the simple forms of the quark and gluon propagators resulted in quantitatively correct estimates for three different sectors of the low-energy particle physics, the glueball and meson spectra as well as the weak decay constants.

\section{References}

[1] R.Alkofer and J. Greensite, J. Phys. G 34 (2007) S3.

[2] H. Leutwyler, Phys. Lett., 96B (1980) 154; Nucl. Phys., B179 (1981) 129.

[3] G.V. Efimov and S.N. Nedelko, Phys. Rev., D51 (1995) 176; Phys. Rev., D54 (1996) 4483.

[4] E. E. Salpeter and H. A. Bethe, Phys. Rev. 84 (1951) 1232.

[5] G.V. Efimov and G. Ganbold, Phys. Rev., D65 (2002) 054012.

[6] G. Ganbold, AIP Conf. Proc., 796 (2005) 127.

[7] G. Ganbold, arXiv:hep-ph/0610399 (2006); SLAC: eConf/C070910 (2007) .

[8] D.V.Shirkov, Th. Math. Phys., 132 (2002) 484; A.V.Nesterenko, Int. J. Mod. Phys., A18 (2003) 5475.

[9] Particle Data Group, W.M.Yao et al., J. Phys., G33 (2006) 1.

[10] C. Bernard et al., PoS LAT2005 (2005) 025.

[11] E. Klempt and A. Zaitsev, Phys. Rep. 454 (2007) 1.

[12] W. Ochs, arXiv:hep-ph/0609207 (2006).

[13] A. Vaccarino and D. Weingarten, Phys. Rev. D60 (1999) 114501.

[14] C.J. Morningstar and M. Peardon, Phys. Rev. D60 (1999) 034509.

[15] Y. Chen et. al., Phys. Rev., D73 (2006) 014516. 